\title{
Primary breast cancer relapse as metastasis to the cervix uteri: A case report
}

\author{
AUDE THOUVENOT $^{1}$, YASMINE BIZET $^{1}$, LAURENT S. BACCAR ${ }^{2}$ and MICHELE LAMURAGLIA MA $^{3,4}$ \\ ${ }^{1}$ Geriatric Unit and ${ }^{2}$ Radiology Department, AP-HP, Louis Mourier Hospital, 92700 Colombes; \\ ${ }^{3}$ Oncology Unit, AP-HP - Hospital Beaujon, 92110 Clichy; ${ }^{4}$ Biomedical Imaging Laboratory (LIB), \\ AP-HP - Hospital Beaujon, University of Sorbonne, CNRS, INSERM, 75006 Paris, France
}

Received March 19, 2018; Accepted May 14, 2018

DOI: $10.3892 / \mathrm{mco} .2018 .1627$

\begin{abstract}
Metastasis of non-gynaecological tumours to the cervix is a rare event, and metastasis from breast cancer is even rarer, with only a limited number of such cases reported in the literature to date. We herein report the case of an 86-year-old female patient who had undergone mastectomy and axillary lymphadenectomy for invasive ductal cell breast carcinoma 2 years prior, followed by adjuvant hormonal therapy with letrozole. During hospitalization for anemia associated with an inflammatory syndrome and abdominal pain with menorrhagia, an abdominal ultrasound examination revealed a suspicious uterine mass with irregular contours and abnormal vascularization with associated increase of the blood level of cancer antigen 15-3 to $34 \mathrm{kU} / 1$. The histological and immunohistochemical analysis of a cervical biopsy sample discover a secondary lesion metastatic from the primary ductal cell breast carcinoma. The metastatic tissue was hormone-negative, which was compatible with disease progression during hormonal therapy. Considering the multiple metastasis, comorbidities, unfavourable performance status and the quick deterioration of the patient's clinical condition, only best supportive care was administered.
\end{abstract}

\section{Introduction}

Breast cancer is the most frequently diagnosed cancer globally and is the leading cause of cancer-related death in women (1). Breast cancer screening programmes and improved treatment of primary tumors have contributed significantly to reducing overall mortality rates. However, clinical management of patients with metastatic progression is much less comprehensively structured. The ovarian metastases are sufficiently uncommon that they may be overlooked in the differential

Correspondence to: Dr Michele Lamuraglia, Oncology Unit, AP-HP - Hospital Beaujon, 100 Boulevard du Général Leclerc, 92110 Clichy, France

E-mail: michele.lamuraglia@aphp.fr

Key words: breast cancer, cervix, endometrium, metastasis diagnosis of genital tract disorders (2). Same metastatic lesions are clearly secondary metastasis of breast cancer in patients with disseminated disease, while other tumors may be confused with primary neoplasms. This latter group represents the tumors with high aggressiveness as metastasis to the cervix uteri $(2,3)$. It is important to identify these secondary neoplasms early for therapeutic considerations. This case report shows the complexities when issues of metastatic disease are considered and the consequent need to differential diagnostic with histology.

\section{Case report}

An 86-year-old female patient was hospitalized in the Geriatric Unit of Louis Mourier Hospital, Colombes, for anemia associated with an inflammatory syndrome and abdominal pain with menorrhagia, in December 2016. The patient was treated with adjuvant hormonal therapy (letrozole), following right mastectomy and axillary lymphadenectomy for invasive ductal cell carcinoma (T2NOM0) of the breast 2 years prior.

An abdominal ultrasound examination revealed a significant uterine mass $(59 \times 40 \mathrm{~mm})$ with irregular contours and abnormal vascularization, with associated discrete increase of the blood level of cancer antigen 15-3 to $34 \mathrm{kU} / 1$ (normal range, $<30 \mathrm{kU} / \mathrm{l})$. The computed tomography (CT) scan confirmed the uterine mass was situated next to the bladder and had irregular endometrium, with multiple para-aortic lymphadenopathies. The morphological assessment was completed by magnetic resonance imaging (MRI), which revealed myometrial encroachment $>50 \%$ on the right parametrium and adnexa, and iliac nodes (Fig. 1). Positron emission tomography (PET)-CT revealed multiple metastases in the sub-diaphragmatic para-aortic lymph nodes, the left hilum and left adrenal gland, with diffuse bone metastases and peritoneal carcinomatosis

A cervical biopsy was performed and the histological and immunohistochemical analysis (CK7-positive, CK20-negative, anti-E-cadherin-positive, hormone receptor-negative and human epidermal growth factor receptor 2-negative) confirmed this cancer as a secondary lesion of primary ductal cell breast carcinoma, but with a negative hormone profile, compatible with disease progression during hormonal therapy. Unfortunately, considering the multiple comorbidities, 


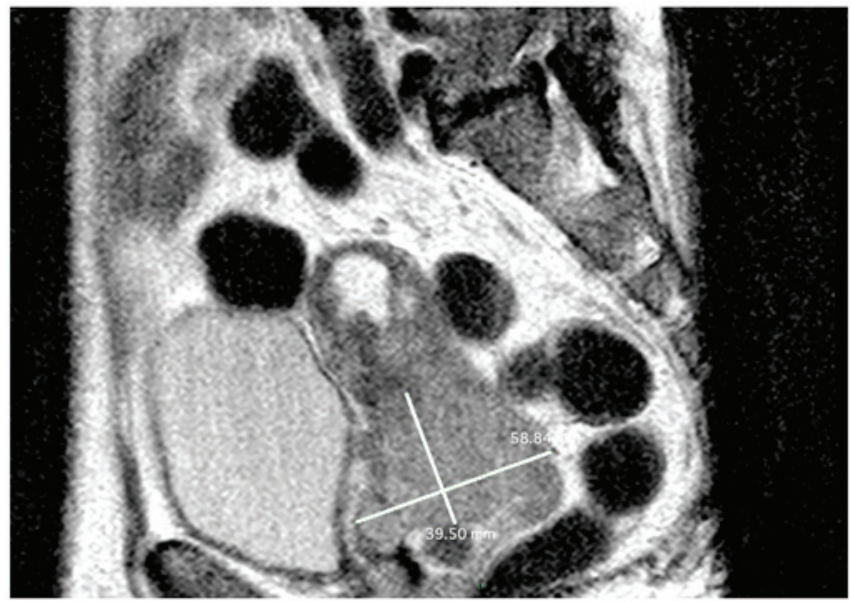

Figure 1. Pelvic magnetic resonance imaging examination revealed a myometrial mass $(59 \times 40 \mathrm{~mm})$ with encroachment of $>50 \%$ on the right parametrium and adnexa.

performance status score of 3 and the quick deterioration of the patient's clinical condition, supportive care was provided until the patient succumbed in July 2017.

\section{Discussion}

Metastasis of non-gynaecological tumours to the cervix is a rare event, and metastasis from breast cancer is even rarer. Only a few such cases have been described in the literature to date, so the true incidence of breast cancer metastasis to cervix is unknown (2-4). Mazur et al in 1984 analysed 325 metastatic female genital neoplasms, only 52 of which were found to be metastases from breast carcinomas, but no cervical metastasis from breast cancer was included (5). More recently, Cumming et al reported that younger women are at a higher risk for breast cancer metastasis to gynaecological sites, than from cervix metastasis (6).

We herein present the case of a breast cancer relapse as cervical metastasis 2 years after surgical treatment for breast cancer, followed by treatment with letrozole. This progression was accompanied by a modification of the metastatic relapse hormonal status. In fact, the negatification of the hormonal status indicates disease progression during adjuvant hormonal therapy.

It is imperative to differentiate breast cancer metastasis from primary carcinoma of the genital tract, but this differentiation may be difficult due to several reasons: Non-specific symptoms at presentation, long disease-free interval, inconclusive radiological findings, and adenocarcinoma histopathology. Imaging (CT or MRI scan) was not sufficient for differential diagnosis in the present case, as the tumour mimicked primary metastatic ovarian carcinoma. Immunohistological analysis, particularly staining with anti-E-cadherin, were required for the differential diagnosis.

It has been reported in the literature that younger women are more likely to develop breast cancer relapse with gynaecological metastasis compared with geriatric patients. Thus, it is imperative that all patients with a history of breast cancer presenting with gynaecological symptoms undergo biopsy of the relapse site. In order to make the right diagnosis, histological analysis is key in differentiating between primary and metastatic disease.

\section{Acknowledgements}

Not applicable.

\section{Funding}

No funding was received.

\section{Availability of data and materials}

The datasets used and/or analyzed during the current study are available from the corresponding author on reasonable request.

\section{Authors' contributions}

AT and YB collected imaging data, performed part of the measurements, analysed data and wrote the manuscript. LB performed the imaging analyses. ML carried out the design of the study and writing of the manuscript. The final version of the manuscript has been read and approved by all authors.

\section{Ethics approval and consent to participate}

The protocol was approved by The Ethics Committee and Institutional Review Board of AP-HP Hospital Louis Mourier (Colombes, France).

\section{Consent for publication}

The patient consent to the publication data and associated image.

\section{Competing interests}

Author declares no conflict of interest.

\section{References}

1. ForouzanfarMH, Foreman KJ, Delossantos AM, Lozano R, Lopez AD, Murray CJ and Naghavi M: Breast and cervical cancer in 187 countries between 1980 and 2010: a systematic analysis. Lancet 378: 1461-1484, 2011.

2. Cruz PT: Mammary carcinoma with metastasis to the cervix uteri. Del Med J 26: 304-305, 1954.

3. Muñoz-Iglesias J, Uña-Gorospe J, Allende-Riera A, De Sequera-Rahola $M$ and Cárdenas-Negro C: Unsuspected uterine metastasis of breast carcinoma diagnosed by $18 \mathrm{~F}-\mathrm{FDG}$ PET/CT. Clin Nucl Med 38: e441-e442, 2013.

4. Benkerroum Z, Babahabib A, Kouach J, Chahdi H, Al Bouzidi A, Moussaoui Rehali D and Dehayni M: Metrorrhagia disclosing a synchronous bilateral breast cancer: Report of a case. Gynécol Obstét Fertil 42: 360-364, 2014 (In French).

5. Mazur MT, Hsueh S and Gersell DJ: Metastases to the female genital tract. Analysis of 325 cases. Cancer 53: 1978-1984, 1984.

6. Cummings MC, Simpson PT, Reid LE, Jayanthan J, Skerman J, Song S, McCart Reed AE, Kutasovic JR, Morey AL, Marquart L, et al: Metastatic progression of breast cancer: Insights from 50 years of autopsies. J Pathol 232: 23-31, 2014. 